\title{
A fractional differential equation with multi-point strip boundary condition involving the Caputo fractional derivative and its Hyers-Ulam stability
}

\author{
Mehboob Alam', Akbar Zada', Ioan-Lucian Popa², Alireza Kheiryan³, Shahram Rezapour ${ }^{3,4^{*}}$ (D) and \\ Mohammed K.A. Kaabar ${ }^{5,6}$
}

"Correspondence:

sh.rezapour@azaruniv.ac.ir;

sh.rezapour@mail.cmuh.org.tw

rezapourshahram@yahoo.ca

${ }^{3}$ Department of Mathematics,

Azarbaijan Shahid Madani

University, Tabriz, Iran

${ }^{4}$ Department of Medical Research,

China Medical University Hospital,

Taichung, Taiwan

Full list of author information is

available at the end of the article

\section{글 Springer}

\begin{abstract}
In this work, we investigate the existence, uniqueness, and stability of fractional differential equation with multi-point integral boundary conditions involving the Caputo fractional derivative. By utilizing the Laplace transform technique, the existence of solution is accomplished. By applying the Bielecki-norm and the classical fixed point theorem, the Ulam stability results of the studied system are presented. An illustrative example is provided at the last part to validate all our obtained theoretical results.
\end{abstract}

MSC: Primary 34A08; secondary 34A12

Keywords: Caputo fractional derivative; Hybrid fractional differential equation and inclusion; Thermostat modeling

\section{Introduction}

In the last few decades, a special consideration has been paid to fractional differential equations (FDEs) due to their wide range applications into real world phenomena (see [1-4]). Various attempts have been made in order to present these phenomena in a superior way and to explore new fractional derivatives with different approaches such as Riemann-Liouville, Caputo, Hadamard, Hilfer-Hadamard, and Grünwald-Letnikov [511]. In fact, FDEs are nonlocal in nature, and they describe many nonlinear phenomena very precisely, so they have a huge impact on different disciplines of science like hydrodynamics, control theory, signal processing, and image processing. More applications in these multidisciplinary sciences can be traced in [12-15]. In literature, there exist many complex differential systems that cannot be solved analytically, and obtaining the solution of such a type of systems is a big challenge to mathematicians; therefore, in such a situation, a solution can be traced through its properties, which are known as qualitative properties. One of the interesting examples is to investigate the unique solution's existence of an elliptic partial differential equation provided that there is a known average value [16].

(c) The Author(s) 2021. This article is licensed under a Creative Commons Attribution 4.0 International License, which permits use, sharing, adaptation, distribution and reproduction in any medium or format, as long as you give appropriate credit to the original author(s) and the source, provide a link to the Creative Commons licence, and indicate if changes were made. The images or other third party material in this article are included in the article's Creative Commons licence, unless indicated otherwise in a credit line to the material. If material is not included in the article's Creative Commons licence and your intended use is not permitted by statutory regulation or exceeds the permitted use, you will need to obtain permission directly from the copyright holder. To view a copy of this licence, visit http://creativecommons.org/licenses/by/4.0/. 
In addition, another interesting example is the nonlinear problem of implicit FDEs with impulsive and integral boundary conditions which was investigated in [17] with the help of Schaefer's fixed point theorem and Banach's contraction principle. In fact, the qualitative properties like the existence and uniqueness theory for the solutions of fractional models with boundary value problem have attracted great attention among the researchers [18-27].

Another remarkable area which has recently received a considerable attention as one of the central topics in mathematical analysis is the stability of FDEs. In 1940, Ulam [28] initiated the stability of functional equations, which was improved by Hyers [29] in 1941 via Banach spaces. That is the reason why this stability is named Hyers-Ulam stability $(\mathcal{H U S})$. After that, Rassias [30] introduced the Hyers-Ulam-Rassias stability (HURS) by generalizing the concept of $\mathcal{H U S}$. Moreover, a number of mathematicians have spread the idea of $\mathcal{H U S}$ to different classes of functional equations [31-33].

Abbas et al. [34] studied the Ulam stability and existence and uniqueness of solutions for the FDE

$$
\left\{\begin{array}{l}
{ }^{H} \mathcal{D}_{1^{+}}^{\alpha, \beta} \mathrm{u}(\sigma)=f(\sigma, \mathrm{u}(\sigma)), \quad \sigma \in[1, T] \\
{ }^{H} \mathcal{I}_{1^{+}}^{\alpha, 1-\gamma} \mathrm{u}(1)=\phi
\end{array}\right.
$$

where $0<\alpha<1,0 \leq \beta \leq 1, \gamma=\alpha+\beta-\alpha \beta, \phi \in \mathbb{R}, T>1,{ }^{H} \mathcal{D}_{1^{+}}^{\alpha, \beta}(\cdot)$ and ${ }^{H} \mathcal{I}_{1^{+}}^{\alpha, 1-\gamma}(\cdot)$ are the Hilfer-Hadamard fractional derivative and the Hadamard fractional integral, respectively.

Chalishajar et al. [35] studied the $\mathcal{H} \mathcal{U S}$ and the existence and uniqueness of solutions of

$$
\begin{cases}{ }^{c} \mathcal{D}_{0^{+}}^{\alpha} \mathrm{u}(\sigma)=\phi_{1}(\sigma, \mathrm{v}(\sigma)), & \alpha \in(1,2], \sigma \in[0,1], \\ { }^{c} \mathcal{D}_{0^{+}}^{\beta} \mathrm{v}(\sigma)=\phi_{2}(\sigma, \mathrm{u}(\sigma)), & \beta \in(1,2], \sigma \in[0,1], \\ \mathfrak{p u}(0)+\mathfrak{q u} \mathrm{u}^{\prime}(0)=\int_{0}^{1} \varphi_{1}(\mathrm{u}(s)) d s, & \mathfrak{p u}(1)+\mathfrak{q u} \mathrm{u}^{\prime}(1)=\int_{0}^{1} \varphi_{2}(\mathrm{u}(s)) d s, \\ \widetilde{\mathfrak{p}} \mathrm{v}(0)+\widetilde{\mathfrak{q}} \mathrm{v}^{\prime}(0)=\int_{0}^{1} \widetilde{\varphi}_{1}(\mathrm{v}(s)) d s, & \widetilde{\mathfrak{p}} \mathrm{v}(1)+\widetilde{\mathfrak{q}} \mathrm{v}^{\prime}(1)=\int_{0}^{1} \widetilde{\varphi}_{2}(\mathrm{v}(s)) d s,\end{cases}
$$

where ${ }^{c} \mathcal{D}_{0^{+}}^{(\cdot)}(\cdot)$ represents the fractional derivative in a Caputo sense, $\mathfrak{p}, \tilde{\mathfrak{p}}>0, \mathfrak{q}, \tilde{\mathfrak{q}} \geq 0$, and $\varphi_{1}, \widetilde{\varphi}_{1}, \varphi_{2}, \widetilde{\varphi}_{2}$ are continuous functions.

In [36], the authors studied the $\mathcal{H U S}$ and $\mathcal{H U} \mathcal{R S}$ of Volterra integro-differential equation as follows:

$$
\left\{\begin{array}{l}
{ }^{H} \mathcal{D}_{0^{+}}^{\alpha, \beta ; \psi} \mathrm{u}(\sigma)=f(\sigma, \mathrm{u}(\sigma))+\int_{0}^{\sigma} \mathcal{K}(\sigma, s, \mathrm{u}(\sigma)) d s, \quad \sigma \in \mathrm{J}=[0, T], \\
\left.\mathcal{I}_{0^{+}}^{1-\gamma} \mathrm{u}(\sigma)\right|_{\sigma=0}=\mathrm{C}
\end{array}\right.
$$

where $f(\sigma, \mathrm{u})$ and $\mathcal{K}(\sigma, s, \mathrm{u})$ are the continuous functions with respect to $\sigma, \mathrm{u}$ on $\mathrm{J} \times \mathbb{R}$, and $\sigma, s$, u on $\mathrm{J} \times \mathrm{J} \times \mathbb{R}$, respectively, $c$ is a given constant, ${ }^{H} \mathcal{D}_{0^{+}}^{\alpha, \beta ; \psi}(\cdot)$ is the Hilfer fractional derivative with $\psi$ such that the fractional orders $\alpha \in(0,1)$ and $\beta \in[0,1]$, and $\mathcal{I}_{0^{+}}^{1-\gamma}(\cdot)$ is the $\psi$-Riemann-Liouville fractional integral such that $\gamma \in[0,1)$.

Dai et al. [37] studied the Caputo fractional derivative along with $\mathcal{H U S}$ and $\mathcal{H U R S}$ for a class of FDEs of the following integral boundary condition:

$$
\left\{\begin{array}{l}
\mathrm{u}^{\prime}(\sigma)+{ }^{c} \mathcal{D}_{0^{+}}^{\alpha} \mathrm{u}(\sigma)=\phi(\sigma, \mathrm{u}(\sigma)), \quad \sigma \in[0,1], \\
\mathrm{u}(1)=\mathcal{I}_{0^{+}}^{\beta} \mathrm{u}(\eta)=\frac{1}{\Gamma(\beta)} \int_{0}^{\eta}(\eta-s)^{\beta-1} \mathrm{u}(s) d s,
\end{array}\right.
$$


where $\alpha \in(0,1), \beta>0, \eta \in(0,1],{ }^{c} \mathcal{D}_{0^{+}}^{\alpha}(\cdot)$ represents the Caputo fractional derivative, $\mathcal{I}_{0^{+}}^{\beta}(\cdot)$ represents the Riemann-Liouville fractional integral, $\mathrm{u} \in \mathrm{C}^{1}[0,1]$, and $\phi:[0,1] \times$ $(-\infty, \infty) \rightarrow(-\infty, \infty)$ is a continuous function.

Motivated by the work introduced in [34-37], in this paper, we study the existence and uniqueness, $\mathcal{H U S}$ and $\mathcal{H U} \mathcal{R S}$ of the accompanying nonlinear FDE including Caputo fractional derivative. The proposed framework is as follows:

$$
\left\{\begin{array}{l}
\mathrm{u}^{\prime \prime}(\sigma)+{ }^{c} \mathcal{D}_{0^{+}}^{\alpha} \mathrm{u}(\sigma)=\phi(\sigma, \mathrm{u}(\sigma)), \quad \sigma \in \mathrm{J}, \\
\mathrm{u}(0)=\sum_{i=0}^{k-2} \epsilon_{i} \mathrm{u}\left(\delta_{i}\right) \quad \mathrm{u}(1)=\mathcal{I}_{0^{+}}^{\beta} \varphi(\eta, \mathrm{u}(\eta))=\frac{1}{\Gamma(\beta)} \int_{0}^{\eta}(\eta-s)^{\beta-1} \varphi(s, \mathrm{u}(s)) d s,
\end{array}\right.
$$

where $\mathrm{J}=[0,1],{ }^{c} \mathcal{D}_{0^{+}}^{\alpha}(\cdot)$ represents the Caputo fractional derivative of order $\alpha$ with $1<\alpha<$ $2, \mathcal{I}_{0^{+}}^{\beta}(\cdot)$ is the Riemann-Liouville fractional integral of order $\beta$ with $\beta>1,0<\eta \leq 1$ is a fixed real number, $\epsilon_{i}, \delta_{i} \geq 0, i=1,2, \ldots, k-2, \mathrm{u} \in \mathrm{C}^{2}[0,1]$, and $\phi, \varphi:[0,1] \times(-\infty, \infty) \rightarrow$ $(-\infty, \infty)$ is a continuous function.

The article is organized as follows: In Sect. 2, we give some basic definitions and theorems associated with both fractional derivatives and integrals. The existence and uniqueness of solution, $\mathcal{H} \mathcal{U S}$, and $\mathcal{H U R S}$ to the considered system (1) are discussed in Sect. 3. A specific example is given in Sect. 4.

\section{Fundamental results}

Let $C^{2}[0,1]$ denote a set of differentiable functions and its derivatives that are continuous on $[0,1]$ with the norm

$$
\|\mathrm{u}\|=\max _{\sigma \in \mathrm{J}}|\mathrm{u}(\sigma)| .
$$

Definition 1 ([38]) For any function $u$, the Riemann-Liouville integral for any noninteger arbitrary order $\alpha$ with $\sigma>0$ is stated as follows:

$$
\mathcal{I}_{0^{+}}^{\alpha} \mathrm{u}(\sigma)=\frac{1}{\Gamma(\alpha)} \int_{0}^{\sigma}(\sigma-s)^{\alpha-1} \mathrm{u}(s) d s .
$$

Definition 2 ([38]) For any function $u$, the Caputo derivative for any noninteger arbitrary order $\alpha \in(p-1, p]$ with $\sigma>0$ is stated as follows:

$$
{ }^{c} \mathcal{D}_{0^{+}}^{\alpha} \mathrm{u}(\sigma)=\frac{1}{\Gamma(p-\alpha)} \int_{0}^{\sigma}(\sigma-s)^{p-\alpha-1} \mathrm{u}^{(p)}(s) d s,
$$

where $p=[\alpha]+1$, and $[\alpha]$ denotes the integer part of $\alpha$. Specifically, if $\mathrm{u}$ is defined on the interval $\mathrm{J}$ and $1<\alpha \leq 2$, then

$$
{ }^{c} \mathcal{D}_{0^{+}}^{\alpha} \mathrm{u}(\sigma)=\frac{1}{\Gamma(2-\alpha)} \int_{0}^{\sigma}(\sigma-s)^{1-\sigma} \mathrm{u}^{\prime \prime}(s) d s .
$$

The Laplace transform of Caputo derivatives is

$$
\mathcal{L}\left\{{ }^{c} \mathcal{D}_{0^{+}}^{\alpha} \mathrm{u}(\sigma)\right\}(s)=s^{\alpha} \mathcal{U}(s)-\sum_{i=0}^{n-1} s^{\alpha-k-1} \mathrm{u}^{(k)}(0), \quad \alpha \in(p-1, p],
$$

where $\mathcal{U}(s)$ is the Laplace transform of the function $\mathrm{u}(\sigma)$. 
Definition 3 The Mittag-Leffler $(\mathcal{M L})$ functions for arbitrary $\varrho \in \mathbb{C}$ are

$$
\begin{aligned}
& \mathrm{E}_{\alpha}(\varrho)=\sum_{i=0}^{\infty} \frac{\varrho^{i}}{\Gamma(\alpha i+1)}, \quad \alpha \in \mathbb{C}, \Re(\alpha)>0, \\
& \mathrm{E}_{\alpha, \beta}(\varrho)=\sum_{i=0}^{\infty} \frac{\varrho^{i}}{\Gamma(\alpha i+\beta)}, \quad \alpha, \beta \in \mathbb{C}, \Re(\alpha), \Re(\beta)>0 .
\end{aligned}
$$

The Laplace transforms of $\mathcal{M L}$ functions are defined by

$$
\begin{aligned}
& \mathcal{L}\left\{\mathrm{E}_{\alpha, 1}\left( \pm \lambda \sigma^{\alpha}\right)\right\}(s)=\frac{s^{\alpha-1}}{\left(s^{\alpha} \mp \lambda\right)}, \quad \Re(\alpha)>0, \lambda \in \mathbb{R}, \\
& \mathcal{L}\left\{\sigma^{\beta-1} \mathrm{E}_{\alpha, \beta}\left( \pm \lambda \sigma^{\alpha}\right)\right\}(s)=\frac{s^{\alpha-\beta}}{\left(s^{\alpha} \mp \lambda\right)}, \quad \Re(\alpha), \Re(\beta)>0, \lambda \in \mathbb{R} .
\end{aligned}
$$

Definition 4 ([39]) A function $u$ is said to be of exponential order if there are constants $\tilde{M}, 入>0$ with

$$
|\mathrm{u}(\sigma)| \leq \tilde{M} e^{\lambda \sigma} \quad \text { for all } \sigma>0
$$

Theorem 5 ([40] Krasnosel'skii fixed point theorem) Let $\Lambda$ be a closed convex and nonempty subset of a Banach space $\mathfrak{X}$. Let $\mathfrak{T}_{1}, \mathfrak{T}_{2}$ be operators such that

- $\left[\mathcal{A}_{1}\right] \mathfrak{T}_{1} \mathfrak{w}+\mathfrak{T}_{2} \mathfrak{x} \in \Lambda$ whenever $\mathfrak{w}, \mathfrak{x} \in \Lambda$;

- $\left[\mathcal{A}_{2}\right] \mathfrak{T}_{1}$ is a completely continuous operator;

- $\left[\mathcal{A}_{3}\right] \mathfrak{T}_{2}$ is a contractive operator.

Then there exists $\mathfrak{y} \in \Lambda$ such that $\mathfrak{y}=\mathfrak{T}_{1} \mathfrak{y}+\mathfrak{T}_{2} \mathfrak{y}$.

Theorem 6 ([41] Generalized Banach's fixed point theorem) Let $(\mathfrak{X}, d)$ be a generalized complete metric space. Assume that $\mathfrak{T}: \mathfrak{X} \rightarrow \mathfrak{X}$ is a strictly contractive operator with Lipschitz constant $\mathfrak{L}<1$. If there exists $\kappa \geq 0$ such that $d\left(\mathfrak{T}^{\kappa+1} \mathfrak{x}, \mathfrak{T}^{\kappa} \mathfrak{x}\right)<\infty$ for some $\mathfrak{x} \in \mathfrak{X}$, then the following propositions hold:

- $\left[\mathcal{A}_{1}\right]$ The sequence $\left\{\mathfrak{T}^{n} \mathfrak{x}\right\}$ converges to a fixed point $\tilde{\mathfrak{x}}$ of $\mathfrak{T}$;

- $\left[\mathcal{A}_{2}\right]$ The unique fixed point of $\mathfrak{T}$ is $\tilde{\mathfrak{x}} \in \tilde{\mathfrak{X}}=\left\{\mathfrak{y} \in \mathfrak{X}: d\left(\mathfrak{T}^{\kappa} \mathfrak{x}, \mathfrak{y}\right)<\infty\right\}$;

- $\left[\mathcal{A}_{3}\right]$ If $\mathfrak{y} \in \tilde{\mathfrak{X}}$, then $d(\mathfrak{y}, \tilde{\mathfrak{x}}) \leq \frac{1}{1-\mathfrak{l}} d(\mathfrak{T} \mathfrak{y}, \mathfrak{y})$.

Theorem 7 ([39]) The Laplace transform of any function $\mathrm{u}(\mathcal{L}\{\mathrm{u}(\sigma)\})$ exists and converges absolutely for $\Re(s)>\lambda$ if $\mathrm{u}$ is of exponential order.

\section{Main results}

Here, we use assumptions for the existence and uniqueness of solution to the considered problem (1) under Krasnosel'skii and generalized Banach fixed point theorems. We also discuss the $\mathcal{H} \mathcal{U S}$ and $\mathcal{H} \mathcal{U R S}$ for the solution of considered system (1). The following hypotheses need to hold for the upcoming results:

- $\left[\mathcal{H}_{1}\right]$ Let $\varphi, \phi: \mathrm{J} \times(-\infty, \infty) \rightarrow(-\infty, \infty)$ be continuous, then there exist constants $\mathcal{Q}_{\varphi}, \mathcal{Q}_{\phi}>0$ such that $\sigma \in \mathrm{J}$ and $\forall \mathfrak{h}_{1}, \mathfrak{h}_{2} \in \mathbb{R}$

$$
\left|\varphi\left(\sigma, \mathfrak{h}_{1}\right)-\varphi\left(\sigma, \mathfrak{h}_{2}\right)\right| \leq \mathcal{Q}_{\varphi}\left|\mathfrak{h}_{1}-\mathfrak{h}_{2}\right|
$$


and

$$
\left|\phi\left(\sigma, \mathfrak{h}_{1}\right)-\phi\left(\sigma, \mathfrak{h}_{2}\right)\right| \leq \mathcal{Q}_{\phi}\left|\mathfrak{h}_{1}-\mathfrak{h}_{2}\right|
$$

- $\left[\mathcal{H}_{2}\right]$ There exist bounded functions $\mathfrak{f}_{1}, \mathfrak{g}_{1}, \mathfrak{f}_{2}, \mathfrak{g}_{2} \in \mathrm{C}^{2}[0,1]$ such that

$$
|\varphi(\sigma, \mathfrak{h}(\sigma))| \leq \mathfrak{f}_{1}(\sigma)+\mathfrak{g}_{1}(\sigma)|\mathfrak{h}(\sigma)|
$$

and $|\phi(\sigma, \mathfrak{h}(\sigma))| \leq \mathfrak{f}_{2}(\sigma)+\mathfrak{g}_{2}(\sigma)|\mathfrak{h}(\sigma)|$ with $\hat{\mathfrak{f}}_{1}=\sup _{\sigma \in \mathrm{J}} \mathfrak{f}_{1}(\sigma), \hat{\mathfrak{g}}_{1}=\sup _{\sigma \in \mathrm{J}} \mathfrak{g}_{1}(\sigma)$, $\hat{\mathfrak{f}}_{2}=\sup _{\sigma \in \mathrm{J}} \mathfrak{f}_{2}(\sigma)$, and $\hat{\mathfrak{g}}_{2}=\sup _{\sigma \in \mathrm{J}} \mathfrak{g}_{2}(\sigma)<1$.

\subsection{Existence and uniqueness}

Here, we examine the existence and uniqueness as follows.

Lemma 8 Let $\mathrm{u}(\sigma) \in \mathrm{C}^{2}[0,1], 1<\alpha<2, \beta>1$. Also, let $\mathrm{g}$ be any continuous function and $0<\eta \leq 1$, then the solution of

$$
\left\{\begin{array}{l}
\mathrm{u}^{\prime \prime}(\sigma)+{ }^{c} \mathcal{D}_{0^{+}}^{\alpha} \mathrm{u}(\sigma)=g(\sigma), \quad \sigma \in[0,1], \\
\mathrm{u}(0)=\sum_{i=0}^{k-2} \epsilon_{i} \mathrm{u}\left(\delta_{i}\right), \quad \mathrm{u}(1)=\mathcal{I}_{0^{+}}^{\beta} \varphi(\eta, \mathrm{u}(\eta))=\frac{1}{\Gamma(\beta)} \int_{0}^{\eta}(\eta-s)^{\beta-1} \varphi(s, \mathrm{u}(s)) d s,
\end{array}\right.
$$

is given by

$$
\begin{aligned}
\mathrm{u}(\sigma)= & \int_{0}^{1} \mathbb{G}(\sigma, s) g(s) d s+\frac{\left(\vartheta_{1} \sigma+\vartheta_{3}\right)}{\Gamma(\beta)} \int_{0}^{\eta}(\eta-s)^{\beta-1} \varphi(s, \mathrm{u}(s)) d s \\
& +\vartheta_{2}(1-\sigma) \sum_{i=0}^{k-2} \epsilon_{i} \int_{0}^{\delta_{i}}\left(\delta_{i}-s\right) \mathrm{E}_{2-\alpha, 2}\left(-\left(\delta_{i}-s\right)^{2-\alpha}\right) g(s) d s
\end{aligned}
$$

where $\mathbb{G}(\sigma, s)$ is given by

$$
\mathbb{G}(\sigma, s)=\left\{\begin{array}{cc}
(\sigma-s) \mathrm{E}_{2-\alpha, 2}\left(-(\sigma-s)^{2-\alpha}\right) & \\
-\left(\vartheta_{1} \sigma+\vartheta_{3}\right)(1-s) \mathrm{E}_{2-\alpha, 2}\left(-(1-s)^{2-\alpha}\right), & 0 \leq s \leq \sigma, \\
-\left(\vartheta_{1} \sigma+\vartheta_{3}\right)(1-s) \mathrm{E}_{2-\alpha, 2}\left(-(1-s)^{2-\alpha}\right), & \sigma \leq s \leq 1,
\end{array}\right.
$$

and

$$
\begin{aligned}
& \vartheta_{1}=\frac{1-\sum_{i=0}^{k-2} \epsilon_{i}}{1-\sum_{i=0}^{k-2} \epsilon_{i}+\sum_{i=0}^{k-2} \epsilon_{i} \delta_{i}}, \\
& \vartheta_{2}=\frac{1}{1-\sum_{i=0}^{k-2} \epsilon_{i}+\sum_{i=0}^{k-2} \epsilon_{i} \delta_{i}}, \\
& \vartheta_{3}=\frac{\sum_{i=0}^{k-2} \epsilon_{i} \delta_{i}}{1-\sum_{i=0}^{k-2} \epsilon_{i}+\sum_{i=0}^{k-2} \epsilon_{i} \delta_{i}} .
\end{aligned}
$$


Proof Since $\mathrm{u}(\sigma) \in \mathrm{C}^{2}[0,1], \mathrm{u}(\sigma)$ and ${ }^{c} \mathcal{D}_{0^{+}}^{\alpha} \mathrm{u}(\sigma)$ are bounded. We have that $\mathrm{u}^{\prime \prime}$ and ${ }^{c} \mathcal{D}_{0^{+}}^{\alpha}$ are of exponential order, where $\sigma \in \mathrm{J}$. Taking the Laplace transform of (4), by (2), we acquire

$$
\begin{aligned}
& s^{2} \mathcal{U}(s)-s \mathrm{u}(0)-\mathrm{u}^{\prime}(0)+s^{\alpha} \mathcal{U}(s)-s^{\alpha-1} \mathrm{u}(0)-s^{\alpha-2} \mathrm{u}^{\prime}(0)=\mathcal{G}(s), \\
& \mathcal{U}(s)=\frac{s^{-\alpha}}{1+s^{2-\alpha}} \mathcal{G}(s)+\frac{1}{s} \mathrm{u}(0)+\frac{1}{s^{2}} \mathrm{u}(0) .
\end{aligned}
$$

Applying the inverse Laplace transform, by (3), we have

$$
\mathrm{u}(\sigma)=\int_{0}^{\sigma}(\sigma-s) \mathrm{E}_{2-\alpha, 2}\left(-(\sigma-s)^{2-\alpha}\right) g(s) d s+\mathrm{u}(0)+\sigma \mathrm{u}^{\prime}(0) .
$$

Further, we acquire

$$
\begin{aligned}
& \sum_{i=0}^{k-2} \epsilon_{i} \mathrm{u}\left(\delta_{i}\right)=\sum_{i=0}^{k-2} \epsilon_{i} \int_{0}^{\delta_{i}}\left(\delta_{i}-s\right) \mathrm{E}_{2-\alpha, 2}\left(-\left(\delta_{i}-s\right)^{2-\alpha}\right) \mathrm{g}(s) d s+\sum_{i=0}^{k-2} \epsilon_{i} \mathrm{u}(0)+\sum_{i=0}^{k-2} \epsilon_{i} \delta_{i} \mathrm{u}^{\prime}(0), \\
& \sum_{i=0}^{k-2} \epsilon_{i} \mathrm{u}\left(\delta_{i}\right)=\sum_{i=0}^{k-2} \epsilon_{i} \int_{0}^{\delta_{i}}\left(\delta_{i}-s\right) \mathrm{E}_{2-\alpha, 2}\left(-\left(\delta_{i}-s\right)^{2-\alpha}\right) g(s) d s+\sum_{i=0}^{k-2} \epsilon_{i} \mathrm{u}(0)+\sum_{i=0}^{k-2} \epsilon_{i} \delta_{i} \mathrm{u}^{\prime}(0) .
\end{aligned}
$$

Applying the boundary conditions, we get

$$
\begin{aligned}
\mathrm{u}(0)= & \vartheta_{2} \sum_{i=0}^{k-2} \epsilon_{i} \int_{0}^{\delta_{i}}\left(\delta_{i}-s\right) \mathrm{E}_{2-\alpha, 2}\left(-\left(\delta_{i}-s\right)^{2-\alpha}\right) \mathrm{g}(s) d s+\frac{\vartheta_{3}}{\Gamma(\beta)} \int_{0}^{\eta}(\eta-s)^{\beta-1} \varphi(s, \mathrm{u}(s)) d s \\
& -\vartheta_{3} \int_{0}^{1}(1-s) \mathrm{E}_{2-\alpha, 2}\left(-(1-s)^{2-\alpha}\right) \mathrm{g}(s) d s
\end{aligned}
$$

and

$$
\begin{aligned}
\mathrm{u}^{\prime}(0)= & -\vartheta_{2} \sum_{i=0}^{k-2} \epsilon_{i} \int_{0}^{\delta_{i}}\left(\delta_{i}-s\right) \mathrm{E}_{2-\alpha, 2}\left(-\left(\delta_{i}-s\right)^{2-\alpha}\right) \mathrm{g}(s) d s \\
& +\frac{\vartheta_{1}}{\Gamma(\beta)} \int_{0}^{\eta}(\eta-s)^{\beta-1} \varphi(s, \mathrm{u}(s)) d s \\
& -\vartheta_{1} \int_{0}^{1}(1-s) \mathrm{E}_{2-\alpha, 2}\left(-(1-s)^{2-\alpha}\right) g(s) d s
\end{aligned}
$$

Thus, substituting (7) and (8) into (6), we deduce that

$$
\begin{aligned}
\mathrm{u}(\sigma)= & \frac{\left(\vartheta_{1} \sigma+\vartheta_{3}\right)}{\Gamma(\beta)} \int_{0}^{\eta}(\eta-s)^{\beta-1} \varphi(s, \mathrm{u}(s)) d s+\int_{0}^{\sigma}(\sigma-s) \mathrm{E}_{2-\alpha, 2}\left(-(\sigma-s)^{2-\alpha}\right) g(s) d s \\
& +\vartheta_{2}(1-\sigma) \sum_{i=0}^{k-2} \epsilon_{i} \int_{0}^{\delta_{i}}\left(\delta_{i}-s\right) \mathrm{E}_{2-\alpha, 2}\left(-\left(\delta_{i}-s\right)^{2-\alpha}\right) g(s) d s \\
& -\left(\vartheta_{1} \sigma+\vartheta_{3}\right) \int_{0}^{1}(1-s) \mathrm{E}_{2-\alpha, 2}\left(-(1-s)^{2-\alpha}\right) g(s) d s \\
= & \frac{\left(\vartheta_{1} \sigma+\vartheta_{3}\right)}{\Gamma(\beta)} \int_{0}^{\eta}(\eta-s)^{\beta-1} \varphi(s, \mathrm{u}(s)) d s
\end{aligned}
$$




$$
\begin{aligned}
& +\vartheta_{2}(1-\sigma) \sum_{i=0}^{k-2} \epsilon_{i} \int_{0}^{\delta_{i}}\left(\delta_{i}-s\right) \mathrm{E}_{2-\alpha, 2}\left(-\left(\delta_{i}-s\right)^{2-\alpha}\right) \mathrm{g}(s) d s \\
& -\left(\vartheta_{1} \sigma+\vartheta_{3}\right) \int_{\sigma}^{1}(1-s) \mathrm{E}_{2-\alpha, 2}\left(-(1-s)^{2-\alpha}\right) \mathrm{g}(s) d s \\
& +\int_{0}^{\sigma}\left((\sigma-s) \mathrm{E}_{2-\alpha, 2}\left(-(\sigma-s)^{2-\alpha}\right)\right. \\
& \left.-\left(\vartheta_{1} \sigma+\vartheta_{3}\right)(1-s) \mathrm{E}_{2-\alpha, 2}\left(-(1-s)^{2-\alpha}\right)\right) g(s) d s \\
& =\int_{0}^{1} \mathbb{G}(\sigma, s) g(s) d s+\frac{\left(\vartheta_{1} \sigma+\vartheta_{3}\right)}{\Gamma(\beta)} \int_{0}^{\eta}(\eta-s)^{\beta-1} \varphi(s, \mathrm{u}(s)) d s \\
& +\vartheta_{2}(1-\sigma) \sum_{i=0}^{k-2} \epsilon_{i} \int_{0}^{\delta_{i}}\left(\delta_{i}-s\right) \mathrm{E}_{2-\alpha, 2}\left(-\left(\delta_{i}-s\right)^{2-\alpha}\right) \mathrm{g}(s) d s .
\end{aligned}
$$

Hence, the proof is completed.

Remark 1 Using the definition of $\mathcal{M L}$ function, we obtain

$$
\int_{0}^{\sigma}(\sigma-s) \mathrm{E}_{2-\alpha, 2}\left(-(\sigma-s)^{2-\alpha}\right) d s=\sigma^{3} \mathrm{E}_{2-\alpha, 4}\left(-\sigma^{2-\alpha}\right), \quad(\sigma \in \mathrm{J}, 1<\alpha<2),
$$

this implies that the series is convergent. Thus, there exists a constant $E_{2-\alpha, 4}>0$ such that

$$
\left|\int_{0}^{\sigma}(\sigma-s) \mathrm{E}_{2-\alpha, 2}\left(-(\sigma-s)^{2-\alpha}\right) d s\right| \leq\left|\mathrm{E}_{2-\alpha, 4}\left(-\sigma^{2-\alpha}\right)\right| \leq \mathrm{E}_{2-\alpha, 4} .
$$

Moreover, by the continuity of $\mathcal{M L}$ function and (5), there exists a constant $\aleph>0$ such that

$$
\int_{0}^{\sigma}|\mathbb{G}(\sigma, s)| d s \leq \aleph, \quad \sigma \in \mathrm{J}
$$

Theorem 9 Under hypotheses $\left[\mathcal{H}_{1}\right]-\left[\mathcal{H}_{2}\right]$ and the inequality

$$
\frac{\left(\vartheta_{1}+\vartheta_{3}\right) \eta^{\beta} \mathcal{Q}_{\varphi}}{\Gamma(\beta+1)}+\aleph \mathcal{Q}_{\phi}<1
$$

the given system (1) has a unique solution.

Proof Using Lemma 8, the corresponding system (1) has the following solution:

$$
\begin{aligned}
\mathrm{u}(\sigma)= & \int_{0}^{1} \mathbb{G}(\sigma, s) \phi(s, \mathrm{u}(s)) d s+\frac{\left(\vartheta_{1} \sigma+\vartheta_{3}\right)}{\Gamma(\beta)} \int_{0}^{\eta}(\eta-s)^{\beta-1} \varphi(s, \mathrm{u}(s)) d s \\
& +\vartheta_{2}(1-\sigma) \sum_{i=0}^{k-2} \epsilon_{i} \int_{0}^{\delta_{i}}\left(\delta_{i}-s\right) \mathrm{E}_{2-\alpha, 2}\left(-\left(\delta_{i}-s\right)^{2-\alpha}\right) \phi(s, \mathrm{u}(s)) d s,
\end{aligned}
$$

where $\mathbb{G}(\sigma, s)$ is given by (5). 
Consider the operator $\mathfrak{T}$ defined on $\mathrm{C}^{2}[0,1]$ by

$$
\begin{aligned}
(\mathfrak{T u})(\sigma)= & \int_{0}^{1} \mathbb{G}(\sigma, s) \phi(s, \mathrm{u}(s)) d s+\frac{\left(\vartheta_{1} \sigma+\vartheta_{3}\right)}{\Gamma(\beta)} \int_{0}^{\eta}(\eta-s)^{\beta-1} \varphi(s, \mathrm{u}(s)) d s \\
& +\vartheta_{2}(1-\sigma) \sum_{i=0}^{k-2} \epsilon_{i} \int_{0}^{\delta_{i}}\left(\delta_{i}-s\right) \mathrm{E}_{2-\alpha, 2}\left(-\left(\delta_{i}-s\right)^{2-\alpha}\right) \phi(s, \mathrm{u}(s)) d s .
\end{aligned}
$$

Thus, we have $\mathfrak{T} u \in C^{2}[0,1]$ for any $u \in C^{2}[0,1]$. This proves that $\mathfrak{T}$ maps $C^{2}[0,1]$ into itself.

Let $\mathcal{B}=\left\{u \in C^{2}[0,1]:\|u\|<\epsilon\right\}$ and choose

$$
\epsilon>\frac{\left(\frac{\left(\vartheta_{1}+\vartheta_{3}\right) \eta^{\beta}}{\Gamma(\beta+1)} \hat{\mathfrak{f}}_{1}+\aleph \hat{\mathfrak{f}}_{2}\right)}{1-\left(\frac{\left.\vartheta_{1}+\vartheta_{3}\right) \eta^{\beta}}{\Gamma(\beta+1)} \hat{\mathfrak{g}}_{1}+\aleph \hat{\mathfrak{g}}_{2}\right)} .
$$

From (9), using $\left[\mathcal{H}_{2}\right]$ and for $u \in \mathcal{B}$, we get

$$
\begin{aligned}
|(\mathfrak{T u})(\sigma)| \leq & \int_{0}^{1}|\mathbb{G}(\sigma, s)||\phi(s, \mathrm{u}(s))| d s+\frac{\left|\left(\vartheta_{1} \sigma+\vartheta_{3}\right)\right|}{\Gamma(\beta)} \int_{0}^{\eta}(\eta-s)^{\beta-1}|\varphi(s, \mathrm{u}(s))| d s \\
& +\vartheta_{2}|(1-\sigma)| \sum_{i=0}^{k-2} \epsilon_{i} \int_{0}^{\delta_{i}}\left|\left(\delta_{i}-s\right) \mathrm{E}_{2-\alpha, 2}\left(-\left(\delta_{i}-s\right)^{2-\alpha}\right)\right||\phi(s, \mathrm{u}(s))| d s \\
\leq & \frac{\left(\vartheta_{1}+\vartheta_{3}\right) \eta^{\beta}}{\Gamma(\beta+1)}\left(\hat{\mathfrak{f}}_{1}+\hat{\mathfrak{g}}_{1}\|\mathrm{u}\|\right)+\aleph\left(\hat{\mathfrak{f}}_{2}+\hat{\mathfrak{g}}_{2}\|\mathrm{u}\|\right) \\
= & \left(\frac{\left(\vartheta_{1}+\vartheta_{3}\right) \eta^{\beta}}{\Gamma(\beta+1)} \hat{\mathfrak{f}}_{1}+\aleph \hat{\mathfrak{f}}_{2}\right)+\left(\frac{\left(\vartheta_{1}+\vartheta_{3}\right) \eta^{\beta}}{\Gamma(\beta+1)} \hat{\mathfrak{g}}_{1}+\aleph \hat{\mathfrak{g}}_{2}\right)\|\mathrm{u}\| \\
\quad \leq & \left(\frac{\left(\vartheta_{1}+\vartheta_{3}\right) \eta^{\beta}}{\Gamma(\beta+1)} \hat{\mathfrak{f}}_{1}+\aleph \hat{\mathfrak{f}}_{2}\right)+\left(\frac{\left(\vartheta_{1}+\vartheta_{3}\right) \eta^{\beta}}{\Gamma(\beta+1)} \hat{\mathfrak{g}}_{1}+\aleph \hat{\mathfrak{g}}_{2}\right) \epsilon \leq \epsilon \\
\Longrightarrow \quad & \|\mathfrak{T} u\| \leq \epsilon, \quad \mathrm{u} \in \mathcal{B}^{\prime} .
\end{aligned}
$$

Hence, $\mathfrak{T} \mathcal{B} \subseteq \dot{\mathcal{B}}$.

Now, for any $\mathrm{u}_{1}, \mathrm{u}_{2} \in \mathrm{C}^{2}[0,1]$ and $\sigma \in \mathrm{J}$, from (9), using $\left[\mathcal{H}_{1}\right]$, we have

$$
\begin{aligned}
\left|\left(\mathfrak{T u}_{1}\right)(\sigma)-\left(\mathfrak{T} \mathrm{u}_{2}\right)(\sigma)\right| \leq & \int_{0}^{1}|\mathbb{G}(\sigma, s)|\left|\phi\left(s, \mathrm{u}_{1}(s)\right)-\phi\left(s, \mathrm{u}_{2}(s)\right)\right| d s \\
& +\frac{\left|\left(\vartheta_{1} \sigma+\vartheta_{3}\right)\right|}{\Gamma(\beta)} \int_{0}^{\eta}(\eta-s)^{\beta-1}\left|\varphi\left(s, \mathrm{u}_{1}(s)\right)-\varphi\left(s, \mathrm{u}_{2}(s)\right)\right| d s \\
& +\vartheta_{2}\left|(1-\sigma) \sum_{i=0}^{k-2} \epsilon_{i}\right| \int_{0}^{\delta_{i}}\left|\left(\delta_{i}-s\right) \mathrm{E}_{2-\alpha, 2}\left(-\left(\delta_{i}-s\right)^{2-\alpha}\right)\right| \\
& \times\left|\phi\left(s, \mathrm{u}_{1}(s)\right)-\phi\left(s, \mathrm{u}_{1}(s)\right)\right| d s \\
\leq & \frac{\left(\vartheta_{1}+\vartheta_{3}\right) \eta^{\beta} \mathcal{Q}_{\varphi}}{\Gamma(\beta+1)}\left\|\mathrm{u}_{1}-\mathrm{u}_{2}\right\|+\aleph \mathcal{Q}_{\phi}\left\|\mathrm{u}_{1}-\mathrm{u}_{2}\right\| \\
= & \left(\frac{\left(\vartheta_{1}+\vartheta_{3}\right) \eta^{\beta} \mathcal{Q}_{\varphi}}{\Gamma(\beta+1)}+\aleph \mathcal{Q}_{\phi}\right)\left\|\mathrm{u}_{1}-\mathrm{u}_{2}\right\| .
\end{aligned}
$$


As $\frac{\left(\vartheta_{1}+\vartheta_{3}\right) \eta^{\beta} \mathcal{Q}_{\varphi}}{\Gamma(\beta+1)}+\aleph \mathcal{Q}_{\phi}<1$, thus $\mathfrak{T}$ is a contraction mapping. Hence, (1) has a unique solution.

Theorem 10 Under hypotheses $\left[\mathcal{H}_{1}\right]-\left[\mathcal{H}_{2}\right]$ and the inequality

$$
\frac{\left(\vartheta_{1}+\vartheta_{3}\right) \eta^{\beta} \mathcal{Q}_{\varphi}}{\Gamma(\beta+1)}+\aleph \mathcal{Q}_{\phi}<1,
$$

the given system (1) has at least one solution.

Proof Let the operators $\mathfrak{T}_{1}$ and $\mathfrak{T}_{2}$ on $C^{2}[0,1]$ be defined by

$$
\begin{aligned}
\left(\mathfrak{T}_{1} \mathrm{u}\right)(\sigma)= & -\left(\vartheta_{1} \sigma+\vartheta_{3}\right) \int_{\sigma}^{1}(1-s) \mathrm{E}_{2-\alpha, 2}\left(-(1-s)^{2-\alpha}\right) \phi(s, \mathrm{u}(s)) d s, \\
\left(\mathfrak{T}_{2} \mathrm{u}\right)(\sigma)= & \frac{\left(\vartheta_{1} \sigma+\vartheta_{3}\right)}{\Gamma(\beta)} \int_{0}^{\eta}(\eta-s)^{\beta-1} \varphi(s, \mathrm{u}(s)) d s \\
& +\vartheta_{2}(1-\sigma) \sum_{i=0}^{k-2} \epsilon_{i} \int_{0}^{\delta_{i}}\left(\delta_{i}-s\right) \mathrm{E}_{2-\alpha, 2}\left(-\left(\delta_{i}-s\right)^{2-\alpha}\right) \phi(s, \mathrm{u}(s)) d s \\
& +\int_{0}^{\sigma}\left((\sigma-s) \mathrm{E}_{2-\alpha, 2}\left(-(\sigma-s)^{2-\alpha}\right)\right. \\
& \left.-\left(\vartheta_{1} \sigma+\vartheta_{3}\right)(1-s) \mathrm{E}_{2-\alpha, 2}\left(-(1-s)^{2-\alpha}\right)\right) \phi(s, \mathrm{u}(s)) d s .
\end{aligned}
$$

Let $\mathfrak{S}_{\mathfrak{r}}=\left\{u \in C^{2}[0,1]:\|u\| \leq \mathfrak{r}\right\}$ and choose

$$
0<\frac{\left(\frac{\left(\vartheta_{1}+\vartheta_{3}\right) \eta^{\beta}}{\Gamma(\beta+1)} \hat{\mathfrak{f}}_{1}+\aleph \hat{\mathfrak{f}}_{2}\right)}{1-\left(\frac{\left(\vartheta_{1}+\vartheta_{3}\right) \eta^{\beta}}{\Gamma(\beta+1)} \hat{\mathfrak{g}}_{1}+\aleph \mathfrak{\aleph} \hat{\mathfrak{g}}_{2}\right)} \leq \mathfrak{r} .
$$

For any $\mathrm{u}, \mathrm{v} \in \mathfrak{S}_{\mathfrak{r}}$, using $\left[\mathcal{H}_{1}\right]-\left[\mathcal{H}_{2}\right]$, Remark 1 , and the definitions of the operators $\mathfrak{T}_{1}$ and $\mathfrak{T}_{2}$, we get that

$$
\begin{aligned}
\left\|\mathfrak{T}_{1} \mathrm{u}+\mathfrak{T}_{2} \mathrm{v}\right\| \leq & \int_{0}^{1}|\mathbb{G}(\sigma, s)||\phi(s, \mathrm{v}(s))| d s+\frac{\left|\left(\vartheta_{1} \sigma+\vartheta_{3}\right)\right|}{\Gamma(\beta)} \int_{0}^{\eta}(\eta-s)^{\beta-1}|\varphi(s, \mathrm{v}(s))| d s \\
& +\vartheta_{2}|(1-\sigma)| \sum_{i=0}^{k-2} \epsilon_{i} \int_{0}^{\delta_{i}}\left|\left(\delta_{i}-s\right) \mathrm{E}_{2-\alpha, 2}\left(-\left(\delta_{i}-s\right)^{2-\alpha}\right)\right||\phi(s, \mathrm{v}(s))| d s \\
\leq & \frac{\left(\vartheta_{1}+\vartheta_{3}\right) \eta^{\beta}}{\Gamma(\beta+1)}\left(\hat{\mathfrak{f}}_{1}+\hat{\mathfrak{g}}_{1}\|\mathrm{v}\|\right)+\aleph\left(\hat{\mathfrak{f}}_{2}+\hat{\mathfrak{g}}_{2}\|\mathrm{v}\|\right) \\
= & \left(\frac{\left(\vartheta_{1}+\vartheta_{3}\right) \eta^{\beta}}{\Gamma(\beta+1)} \hat{\mathfrak{f}}_{1}+\aleph \hat{\mathfrak{f}}_{2}\right)+\left(\frac{\left(\vartheta_{1}+\vartheta_{3}\right) \eta^{\beta}}{\Gamma(\beta+1)} \hat{\mathfrak{g}}_{1}+\aleph \hat{\mathfrak{g}}_{2}\right)\|\mathrm{v}\| \\
\leq & \left(\frac{\left(\vartheta_{1}+\vartheta_{3}\right) \eta^{\beta}}{\Gamma(\beta+1)} \hat{\mathfrak{f}}_{1}+\aleph \hat{\mathfrak{f}}_{2}\right)+\left(\frac{\left(\vartheta_{1}+\vartheta_{3}\right) \eta^{\beta}}{\Gamma(\beta+1)} \hat{\mathfrak{g}}_{1}+\aleph \hat{\mathfrak{g}}_{2}\right) \mathfrak{r} .
\end{aligned}
$$

Thus, we obtain $\mathfrak{T}_{1} \mathrm{u}+\mathfrak{T}_{2} \mathrm{v} \in \mathfrak{S}_{\mathfrak{r}}$.

Using Theorem 9 , the operator $\mathfrak{T}_{2}$ is a contraction mapping.

By the continuity of $\phi(\sigma, \mathrm{u}(\sigma)), \mathrm{u} \in \mathfrak{S}_{\mathfrak{r}}$, and the two-parameter $\mathcal{M L}$ function, the operator $\mathfrak{T}_{1}$ is continuous. 
Let $\mathrm{u} \in \mathfrak{S}_{\mathfrak{r}}$, from $\left[\mathcal{H}_{2}\right]$ and Remark 1 , we have

$$
\left|\mathfrak{T}_{1} \mathrm{u}(\sigma)\right| \leq \aleph\left(\hat{\mathfrak{f}}_{1}+\hat{\mathfrak{g}}_{1}\|\mathrm{u}\|\right) \leq \dot{\mathcal{C}} .
$$

Hence, $\mathfrak{T}_{1}$ is uniformly bounded on $\mathfrak{S}_{\mathfrak{r}}$.

Let $\mathrm{u} \in \mathfrak{S}_{\mathfrak{r}}$ and $\sigma_{1}, \sigma_{2} \in \mathrm{J}$ such that $\sigma_{1}<\sigma_{2}$,

$$
\begin{aligned}
\left\|\left(\mathfrak{T}_{1} \mathrm{u}\right)\left(\sigma_{1}\right)-\left(\mathfrak{T}_{1} \mathrm{u}\right)\left(\sigma_{2}\right)\right\|= & \|\left(\vartheta_{1} \sigma_{1}+\vartheta_{3}\right) \int_{\sigma_{1}}^{1}(1-s) \mathrm{E}_{2-\alpha, 2}\left(-(1-s)^{2-\alpha}\right) \phi(s, \mathrm{u}(s)) d s \\
& -\left(\vartheta_{1} \sigma_{2}+\vartheta_{3}\right) \int_{\sigma_{2}}^{1}(1-s) \mathrm{E}_{2-\alpha, 2}\left(-(1-s)^{2-\alpha}\right) \phi(s, \mathrm{u}(s)) d s \| \\
= & \| \vartheta_{1} \sigma_{1} \int_{\sigma_{1}}^{1}(1-s) \mathrm{E}_{2-\alpha, 2}\left(-(1-s)^{2-\alpha}\right) \phi(s, \mathrm{u}(s)) d s \\
& -\vartheta_{1} \sigma_{2} \int_{\sigma_{2}}^{1}(1-s) \mathrm{E}_{2-\alpha, 2}\left(-(1-s)^{2-\alpha}\right) \phi(s, \mathrm{u}(s)) d s \\
& +\vartheta_{3} \int_{\sigma_{1}}^{\sigma_{2}}(1-s) \mathrm{E}_{2-\alpha, 2}\left(-(1-s)^{2-\alpha}\right) \phi(s, \mathrm{u}(s)) d s \| \\
\leq & {\left[\vartheta_{1}(1-\theta) \mathrm{E}_{2-\alpha, 2}\left(-(1-\theta)^{2-\alpha}\right)\left(\sigma_{1}\left|1-\sigma_{1}\right|-\sigma_{2}\left|1-\sigma_{2}\right|\right)\right.} \\
& \left.+\vartheta_{3}(1-\theta) \mathrm{E}_{2-\alpha, 2}\left(-(1-\theta)^{2-\alpha}\right)\left|\sigma_{2}-\sigma_{1}\right|\right]\left(\hat{\mathfrak{f}}_{1}+\hat{\mathfrak{g}}_{1}\|\mathrm{u}\|\right),
\end{aligned}
$$

where $\sigma_{1}<\theta<\sigma_{2}$. This implies that

$$
\left\|\left(\mathfrak{T}_{1} \mathrm{u}\right)\left(\sigma_{1}\right)-\left(\mathfrak{T}_{1} \mathrm{u}\right)\left(\sigma_{2}\right)\right\| \rightarrow 0 \quad \text { as } \sigma_{1} \rightarrow \sigma_{2} .
$$

Therefore, $\mathfrak{T}_{1}$ is relatively compact on $\mathfrak{S}_{\mathfrak{r}}$. So, by Theorem 5 and Arzelà-Ascoli theorem, the operator $\mathfrak{T}_{1}$ is compact on $\mathfrak{S}_{r}$. Thus, system (1) has at least one solution on J.

\subsection{Ulam's stability results}

In this subsection, using the Banach fixed point theorem and Bielecki metric, we investigate $\mathcal{H U S}$ and $\mathcal{H U R S}$ results in $\mathrm{C}^{2}[0,1]$ for system (1).

Consider the space $C^{2}[0,1]$ endowed with the Bielecki metric:

$$
d(\mathfrak{p}, \mathfrak{q})=\sup _{\sigma \in \mathrm{J}} \frac{|\mathfrak{p}(\sigma)-\mathfrak{q}(\sigma)|}{\mathfrak{z}(\sigma)}, \quad \mathfrak{p}, \mathfrak{q} \in C^{2}[0,1],
$$

where $\mathfrak{z}: \mathrm{J} \rightarrow(0, \infty)$. Obviously, $\left(\mathrm{C}^{2}[0,1], d\right)$ is a complete metric space.

The following Definitions 11 and 12 are adapted from [36].

Definition 11 If $\hat{\mathfrak{u}}(\sigma)$ is a continuously differentiable function satisfying

$$
\left|\hat{\mathfrak{u}}^{\prime \prime}(\sigma)+{ }^{c} \mathcal{D}_{0^{+}}^{\alpha} \hat{\mathfrak{u}}(\sigma)-\phi(\sigma, \hat{\mathfrak{u}}(\sigma))\right| \leq \rho, \quad \sigma \in \mathrm{J},
$$

where $\rho>0$, and there are a solution $\mathrm{u}(\sigma)$ of $(1)$ and a constant $\wp>0$ independent of $\hat{\mathfrak{u}}(\sigma)$ and $\mathrm{u}(\sigma)$ such that

$$
|\hat{\mathfrak{u}}(\sigma)-\mathrm{u}(\sigma)| \leq \wp \rho, \quad \sigma \in \mathrm{J},
$$

then we say that (1) is $\mathcal{H U S}$. 
Definition 12 If $\hat{\mathfrak{u}}(\sigma)$ is a continuously differentiable function satisfying

$$
\left|\hat{\mathfrak{u}}^{\prime \prime}(\sigma)+{ }^{c} \mathcal{D}_{0^{+}}^{\alpha} \hat{\mathfrak{u}}(\sigma)-\phi(\sigma, \hat{\mathfrak{u}}(\sigma))\right| \leq \mathfrak{z}(\sigma), \quad \sigma \in \mathrm{J},
$$

where $\mathfrak{z}: \mathrm{J} \rightarrow[0, \infty)$ is a continuous function, and there are a solution $\mathrm{u}(\sigma)$ of $(1)$ and a constant $\wp>0$ independent of $\hat{\mathfrak{u}}(\sigma)$ and $\mathrm{u}(\sigma)$ such that

$$
|\hat{\mathfrak{u}}(\sigma)-\mathrm{u}(\sigma)| \leq \wp \mathfrak{z}(\sigma), \quad \sigma \in \mathrm{J},
$$

then we say that (1) is $\mathcal{H} \mathcal{U} \mathcal{R S}$.

Theorem 13 Let hypothesis $\left[\mathcal{H}_{1}\right]$ be satisfied. Moreover, let $\mathfrak{z}: \mathrm{J} \rightarrow(0, \infty)$, and for any constant $0 \leq \mu<1$, we have

$$
\int_{0}^{\sigma}(\sigma-s) \mathrm{E}_{2-\alpha, 2}\left(-(\sigma-s)^{2-\alpha}\right) \mathfrak{z}(s) d s \leq \mu \mathfrak{z}(\sigma) .
$$

If $\hat{\mathfrak{u}} \in \mathrm{C}^{2}[0,1]$ satisfies

$$
\left|\hat{\mathfrak{u}}^{\prime \prime}(\sigma)+{ }^{c} \mathcal{D}_{0^{\alpha}}^{\alpha} \hat{\mathfrak{u}}(\sigma)-\phi(\sigma, \hat{\mathfrak{u}}(\sigma))\right| \leq \mathfrak{z}(\sigma), \quad \sigma \in \mathrm{J},
$$

and $\mathcal{Q}_{\phi} \mu<1$, then there exists a solution $\mathrm{u}(\sigma)$ of $(1)$ in $\mathrm{C}^{2}[0,1]$ such that

$$
|\hat{\mathfrak{u}}(\sigma)-\mathrm{u}(\sigma)| \leq \frac{\mu}{1-\mathcal{Q}_{\phi} \mu} \mathfrak{z}(\sigma), \quad \sigma \in \mathrm{J} .
$$

This implies that under the above conditions, system (1) is $\mathcal{H U R S}$.

Proof Using Lemma 8, the corresponding system (1) has the following solution:

$$
\mathrm{u}(\sigma)=\int_{0}^{\sigma}(\sigma-s) \mathrm{E}_{2-\alpha, 2}\left(-(\sigma-s)^{2-\alpha}\right) \phi(s, \mathrm{u}(s)) d s+\mathrm{u}(0)+\sigma \mathrm{u}^{\prime}(0),
$$

which follows from the proof of (6). We conclude that $u(\sigma)$ satisfies $(1)$ iff $u(\sigma)$ satisfies (14).

Consider the operator $\Omega: C^{2}[0,1] \rightarrow C^{2}[0,1]$ defined by

$$
\begin{aligned}
(\Omega \mathrm{v})(\sigma) & =\int_{0}^{\sigma}(\sigma-s) \mathrm{E}_{2-\alpha, 2}\left(-(\sigma-s)^{2-\alpha}\right) \phi(s, \mathrm{v}(s)) d s+\mathrm{v}(0)+\sigma \mathrm{v}^{\prime}(0), \\
\sigma \in \mathrm{J}, \mathrm{v} & \in \mathrm{C}^{2}[0,1] .
\end{aligned}
$$

Since $\phi$ and the two-parameter $\mathcal{M L}$ function are continuous, this implies that the operator $\Omega$ is continuous.

From (11), for any $\mathfrak{v}, \mathfrak{w} \in \mathrm{C}^{2}[0,1]$, we obtain

$$
\begin{aligned}
d(\Omega \mathfrak{v}, \Omega \mathfrak{w}) & =\sup _{\sigma \in \mathrm{J}} \frac{\left|\int_{0}^{\sigma}(\sigma-s) \mathrm{E}_{2-\alpha, 2}\left(-(\sigma-s)^{2-\alpha}\right)[\phi(s, \mathfrak{v}(s))-\phi(s, \mathfrak{w}(s))] d s\right|}{\mathfrak{z}(\sigma)} \\
& \leq \mathcal{Q}_{\phi} \sup _{\sigma \in \mathrm{J}} \frac{\left|\int_{0}^{\sigma}(\sigma-s) \mathrm{E}_{2-\alpha, 2}\left(-(\sigma-s)^{2-\alpha}\right)\right| \mathfrak{v}(s)-\mathfrak{w}(s)|d s|}{\mathfrak{z}(\sigma)}
\end{aligned}
$$




$$
\begin{aligned}
& =\mathcal{Q}_{\phi} \sup _{\sigma \in J} \frac{\left|\int_{0}^{\sigma}(\sigma-s) \mathrm{E}_{2-\alpha, 2}\left(-(\sigma-s)^{2-\alpha}\right) \mathfrak{z}(\sigma) \frac{|\mathfrak{v}(s)-\mathfrak{w}(s)|}{\mathfrak{z}(\sigma)} d s\right|}{\mathfrak{z}(\sigma)} \\
& \leq \mathcal{Q}_{\phi} \mu d(\mathfrak{v}, \mathfrak{w}) .
\end{aligned}
$$

Since $\mathcal{Q}_{\phi} \mu<1$, the operator $\Omega$ is strictly contractive.

Also, let $\hat{\mathfrak{u}} \in \mathrm{C}^{2}[0,1]$ satisfy (12). Then, we get that $\hat{\mathfrak{u}}$ satisfies the following inequality:

$$
\begin{aligned}
& \left|\hat{\mathfrak{u}}(\sigma)-\hat{\mathfrak{u}}(0)-\sigma \hat{\mathfrak{u}}^{\prime}(0)-\int_{0}^{\sigma}(\sigma-s) \mathrm{E}_{2-\alpha, 2}\left(-(\sigma-s)^{2-\alpha}\right) \phi(s, \hat{\mathfrak{u}}(s)) d s\right| \\
& \quad \leq\left|\int_{0}^{\sigma}(\sigma-s) \mathrm{E}_{2-\alpha, 2}\left(-(\sigma-s)^{2-\alpha}\right) \mathfrak{z}(s) d s\right| .
\end{aligned}
$$

Using (11), (15), and the definition of the operator $\Omega$, we get

$$
\begin{aligned}
|(\Omega \hat{\mathfrak{u}})(\sigma)-\hat{\mathfrak{u}}(\sigma)| & =\left|\hat{\mathfrak{u}}(0)+\sigma \hat{\mathfrak{u}}^{\prime}(0)+\int_{0}^{\sigma}(\sigma-s) \mathrm{E}_{2-\alpha, 2}\left(-(\sigma-s)^{2-\alpha}\right) \phi(s, \hat{\mathfrak{u}}(s)) d s-\hat{\mathfrak{u}}(\sigma)\right| \\
& \leq\left|\int_{0}^{\sigma}(\sigma-s) \mathrm{E}_{2-\alpha, 2}\left(-(\sigma-s)^{2-\alpha}\right) \mathfrak{z}(s) d s\right| \leq \mu \mathfrak{z}(\sigma) .
\end{aligned}
$$

Therefore, we conclude that

$$
d(\Omega \hat{\mathfrak{u}}, \hat{\mathfrak{u}}) \leq \mu<\infty, \quad 0 \leq \mu<1 .
$$

Using $\left[\mathcal{A}_{2}\right]$ of Theorem 6 , there exists an element

$$
\mathrm{u} \in \tilde{\mathrm{C}}[0,1]=\left\{y \in \mathrm{C}^{2}[0,1]: d(\Omega \hat{u}, y)<\infty\right\}
$$

such that $\Omega u=u$ or, likewise,

$$
\mathrm{u}(\sigma)=\int_{0}^{\sigma}(\sigma-s) \mathrm{E}_{2-\alpha, 2}\left(-(\sigma-s)^{2-\alpha}\right) \phi(s, \mathrm{u}(s)) d s+\mathrm{u}(0)+\sigma \mathrm{u}^{\prime}(0) .
$$

Since (14) is the likewise integral equation of $(1)$, so $\mathrm{u}(\sigma)$ is a solution of (1). Also, using $\left[\mathcal{A}_{3}\right]$ of heorem 6 and (16), we have

$$
d(\hat{\mathfrak{u}}, \mathrm{u}) \leq \frac{1}{1-\mathcal{Q}_{\phi} \mu} d(\Omega \hat{\mathfrak{u}}, \hat{\mathfrak{u}}) \leq \frac{\mu}{1-\mathcal{Q}_{\phi} \mu} .
$$

By the definition of $d$, we get that (13) holds.

Theorem 14 Let hypothesis $\left[\mathcal{H}_{1}\right]$ be satisfied, and let $\mathfrak{z}: \mathrm{J} \rightarrow(0, \infty)$, and for any constant $0 \leq \mu<1$, we have

$$
\int_{0}^{\sigma}(\sigma-s) \mathrm{E}_{2-\alpha, 2}\left(-(\sigma-s)^{2-\alpha}\right) \mathfrak{z}(s) d s \leq \mu \mathfrak{z}(\sigma)
$$

and $\mathcal{Q}_{\phi} \mu<1$. If $\hat{\mathfrak{u}} \in \mathrm{C}^{2}[0,1]$ satisfies

$$
\left|\hat{\mathfrak{u}}^{\prime \prime}(\sigma)+{ }^{c} \mathcal{D}_{0^{+}}^{\alpha} \hat{\mathfrak{u}}(\sigma)-\phi(\sigma, \hat{\mathfrak{u}}(\sigma))\right| \leq \rho, \quad \sigma \in \mathrm{J},
$$


where $\rho>0$, then there exists a solution $\mathrm{u}(\sigma)$ of $(1)$ in $\mathrm{C}^{2}[0,1]$ such that

$$
|\hat{\mathfrak{u}}(\sigma)-\mathrm{u}(\sigma)| \leq \frac{\mathfrak{z}(1) \rho \mathrm{E}_{2-\alpha, 4}}{\left(1-\mathcal{Q}_{\phi} \mu\right) \mathfrak{z}(0)}, \quad \sigma \in \mathrm{J}
$$

This implies that under the above conditions, system (1) is $\mathcal{H U S}$.

Proof The first segment of the proof is obtained by performing the same steps as in Theorem 13 . Let the operator $\Omega: C^{2}[0,1] \rightarrow C^{2}[0,1]$ be defined by

$$
\begin{aligned}
& (\Omega \mathrm{v})(\sigma)=\int_{0}^{\sigma}(\sigma-s) \mathrm{E}_{2-\alpha, 2}\left(-(\sigma-s)^{2-\alpha}\right) \phi(s, \mathrm{v}(s)) d s+\mathrm{v}(0)+\sigma \mathrm{v}^{\prime}(0), \\
& \sigma \in \mathrm{J}, \mathrm{v} \in \mathrm{C}^{2}[0,1] .
\end{aligned}
$$

For any $\mathfrak{v}, \mathfrak{w} \in \mathrm{C}^{2}[0,1]$, we have

$$
d(\Omega \mathfrak{v}, \Omega \mathfrak{w}) \leq \mathcal{Q}_{\phi} \mu d(\mathfrak{v}, \mathfrak{w})
$$

Since $\mathcal{Q}_{\phi} \mu<1$, from Theorem 13, this implies that the operator $\Omega$ is strictly contractive in $\left(C^{2}[0,1], d\right)$.

Suppose that $\hat{\mathfrak{u}} \in \mathrm{C}^{2}[0,1]$ satisfies (18). Using Remark 1 , we get

$$
\begin{aligned}
& \left|\hat{\mathfrak{u}}(\sigma)-\hat{\mathfrak{u}}(0)-\sigma \hat{\mathfrak{u}}^{\prime}(0)-\int_{0}^{\sigma}(\sigma-s) \mathrm{E}_{2-\alpha, 2}\left(-(\sigma-s)^{2-\alpha}\right) \phi(s, \hat{\mathfrak{u}}(s)) d s\right| \\
& \quad \leq \rho\left|\int_{0}^{\sigma}(\sigma-s) \mathrm{E}_{2-\alpha, 2}\left(-(\sigma-s)^{2-\alpha}\right) d s\right| \leq \rho \mathrm{E}_{2-\alpha, 4}, \quad \sigma \in \mathrm{J} .
\end{aligned}
$$

Now, by the definition of the operator $\Omega$, we get

$$
\begin{aligned}
|(\Omega \hat{\mathfrak{u}})(\sigma)-\hat{\mathfrak{u}}(\sigma)| & =\left|\hat{\mathfrak{u}}(0)+\sigma \hat{\mathfrak{u}}^{\prime}(0)+\int_{0}^{\sigma}(\sigma-s) \mathrm{E}_{2-\alpha, 2}\left(-(\sigma-s)^{2-\alpha}\right) \phi(s, \hat{\mathfrak{u}}(s)) d s-\hat{\mathfrak{u}}(\sigma)\right| \\
& \leq \rho \mathrm{E}_{2-\alpha, 4}, \quad \sigma \in \mathrm{J} .
\end{aligned}
$$

Since $\mathfrak{z}$ is a nondecreasing positive function, we have

$$
d(\Omega \hat{\mathfrak{u}}, \hat{\mathfrak{u}})=\sup _{\sigma \in J} \frac{|\Omega \hat{\mathfrak{u}}(\sigma)-\hat{\mathfrak{u}}(\sigma)|}{\mathfrak{z}(\sigma)} \leq \frac{\rho \mathrm{E}_{2-\alpha, 4}}{\mathfrak{z}(0)}<\infty .
$$

Using $\left[\mathcal{A}_{2}\right]$ of Theorem 6 , there exists an element

$$
\mathrm{u} \in \tilde{\mathrm{C}}[0,1]=\left\{y \in \mathrm{C}^{2}[0,1]: d(\Omega \hat{u}, y)<\infty\right\}
$$

such that $\Omega u=u$ or, likewise,

$$
\mathrm{u}(\sigma)=\int_{0}^{\sigma}(\sigma-s) \mathrm{E}_{2-\alpha, 2}\left(-(\sigma-s)^{2-\alpha}\right) \phi(s, \mathrm{u}(s)) d s+\mathrm{u}(0)+\sigma \mathrm{u}^{\prime}(0)
$$

which implies $u(\sigma)$ is a solution of (1). 
Thus, from $\left[\mathcal{A}_{3}\right]$ of Theorem 6 and (20), it follows that

$$
d(\hat{\mathfrak{u}}, \mathrm{u}) \leq \frac{1}{1-\mathcal{Q}_{\phi} \mu} \sup _{\sigma \in \mathrm{J}} \frac{|\Omega \hat{\mathfrak{u}}(\sigma)-\hat{\mathfrak{u}}(\sigma)|}{\mathfrak{z}(\sigma)} \leq \frac{1}{1-\mathcal{Q}_{\phi} \mu} \frac{\rho \mathrm{E}_{2-\alpha, 4}}{\mathfrak{z}(0)} .
$$

By the definition of $d$, we get that

$$
|\hat{\mathfrak{u}}(\sigma)-\mathrm{u}(\sigma)| \leq \frac{\mathfrak{z}(\sigma) \rho \mathrm{E}_{2-\alpha, 4}}{\left(1-\mathcal{Q}_{\phi} \mu\right) \mathfrak{z}(0)}, \quad \sigma \in \mathrm{J}
$$

Therefore, (19) follows directly from (21).

\section{Illustrative example}

Here, we provide an adequate problem to testify our results.

Example 1 Let

$$
\left\{\begin{array}{l}
\mathrm{u}^{\prime \prime}(\sigma)+{ }^{c} \mathcal{D}_{0^{+}}^{\frac{3}{2}} \mathrm{u}(\sigma)=\frac{1}{\left(8+\sigma^{2}\right)(1+|\mathrm{u}(\sigma)|)}, \quad \sigma \in \mathrm{J}, \\
\mathrm{u}(0)=\sum_{i=0}^{4} \epsilon_{i} \mathrm{u}\left(\delta_{i}\right) \quad \mathrm{u}(1)=\frac{1}{\Gamma\left(\frac{3}{2}\right)} \int_{0}^{\frac{1}{4}}\left(\frac{1}{4}-s\right)^{\frac{1}{2}} \frac{s^{2}+\mathrm{u}(s)}{60} d s,
\end{array}\right.
$$

where $\epsilon_{0}=\frac{9}{40}, \epsilon_{1}=\frac{47}{200}, \epsilon_{2}=\frac{49}{200}, \epsilon_{3}=\frac{51}{200}, \epsilon_{4}=\frac{53}{200}, \delta_{0}=\frac{21}{40}, \delta_{1}=\frac{107}{200}, \delta_{2}=\frac{109}{200}, \delta_{3}=\frac{111}{200}$, $\delta_{4}=\frac{113}{200}$, and $u(\sigma) \in C^{2}[0,1]$.

From system (1), we see that

$$
\begin{aligned}
& \alpha=\frac{3}{2}, \quad \beta=\frac{3}{2}, \quad \eta=\frac{1}{4}, \\
& \phi(\sigma, \mathrm{u}(\sigma))=\frac{1}{\left(8+\sigma^{2}\right)(1+|\mathrm{u}(\sigma)|)}, \quad \varphi(\sigma, \mathrm{u}(\sigma))=\frac{\sigma^{2}+\mathrm{u}(\sigma)}{60} .
\end{aligned}
$$

Now, for all $\mathfrak{h}_{1}, \mathfrak{h}_{2} \in \mathbb{R}$ and $\sigma \in \mathrm{J}$, we have

$$
\left|\varphi\left(\sigma, \mathfrak{h}_{1}\right)-\varphi\left(\sigma, \mathfrak{h}_{2}\right)\right| \leq \frac{1}{8}\left|\mathfrak{h}_{1}-\mathfrak{h}_{2}\right|
$$

and

$$
\left|\phi\left(\sigma, \mathfrak{h}_{1}\right)-\phi\left(\sigma, \mathfrak{h}_{2}\right)\right| \leq \frac{1}{60}\left|\mathfrak{h}_{1}-\mathfrak{h}_{2}\right|
$$

These satisfy condition $\left[\mathcal{H}_{1}\right]$ with $\mathcal{Q}_{\varphi}=\frac{1}{8}$ and $\mathcal{Q}_{\phi}=\frac{1}{60}$.

Further, by Remark 1, we have

$$
\frac{\left(\vartheta_{1}+\vartheta_{3}\right) \eta^{\beta} \mathcal{Q}_{\varphi}}{\Gamma(\beta+1)}+\aleph \mathcal{Q}_{\phi}=\frac{\left(-\frac{600}{1183}+\frac{1783}{1183}\right) \frac{1}{4}^{\frac{3}{2}} \frac{1}{8}}{\Gamma\left(\frac{5}{2}\right)}+\frac{1}{60}=\frac{1}{48 \sqrt{\pi}}+\frac{1}{60}<1 .
$$

From Theorems 9 and 10, system (1) has a unique solution.

Next, we check the $\mathcal{H} \mathcal{U S}$ and $\mathcal{H} \mathcal{U} \mathcal{R} \mathcal{S}$ for (22).

Letting $\mathfrak{z}(\sigma)=e^{\sigma}$, by Remark 1 , we obtain

$$
\int_{0}^{\sigma}(\sigma-s) \mathrm{E}_{\frac{1}{2}, 2}\left(-(\sigma-s)^{\frac{1}{2}}\right) e^{s} d s<0.56 e^{\sigma}, \quad \sigma \in \mathrm{J} .
$$

Thus, $\mathfrak{z}(\sigma)=e^{\sigma}$ satisfies (11) with $\mu=0.56$ and $\mathcal{Q}_{\phi} \mu=\frac{7}{750}<1$. 


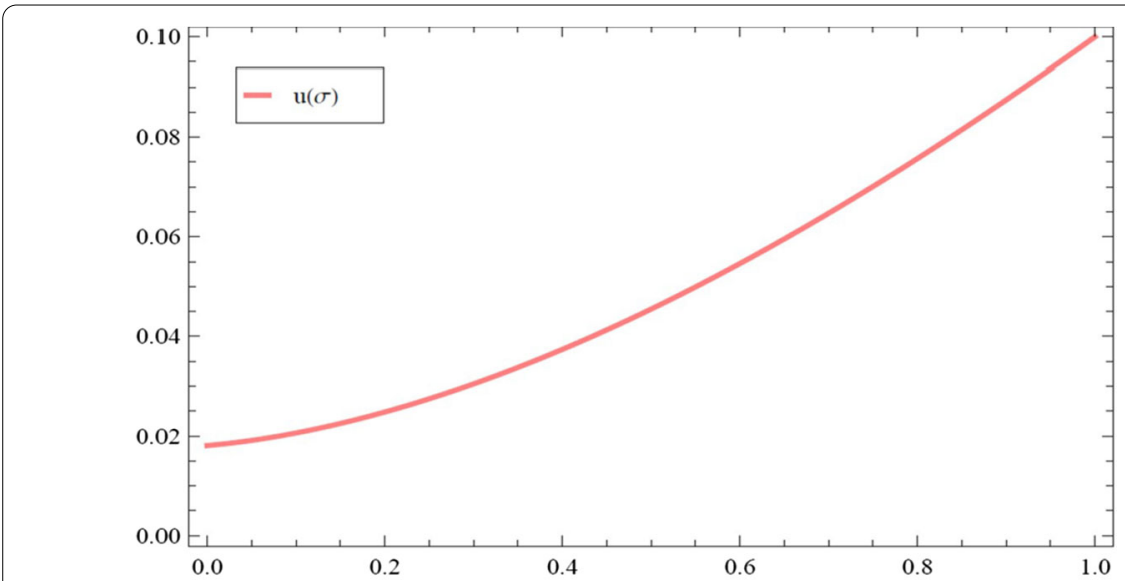

Figure 1 The solution $\mathrm{u}(\sigma)$ of (22) with the given boundary conditions

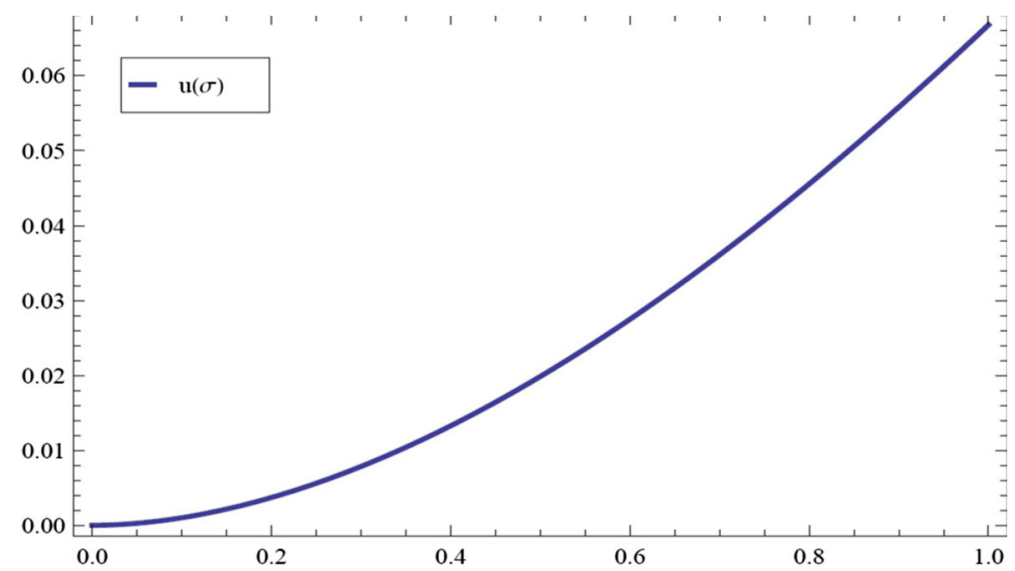

Figure 2 The solution $\mathrm{u}(\sigma)$ of (22) with the initial condition $\mathrm{u}(0)=\mathrm{u}^{\prime}(0)=0$

Hence, from Theorems 13 and 14, the given system (22) is $\mathcal{H U S}$ and $\mathcal{H U R S}$. The $\mathcal{H U S}$ and $\mathcal{H U R S}$ of (22) do not depend on the initial value condition. The solution $u(\sigma)$ of (22) with the given boundary conditions and initial value conditions $u(0)=0$ and $u^{\prime}(0)=0$ is shown in Figs. 1 and 2, respectively.

Now, we consider $v \in \mathrm{C}^{2}[0,1]$ as the solution of the following FDE:

$$
\left\{\begin{array}{l}
\mathrm{v}^{\prime \prime}(\sigma)+{ }^{c} \mathcal{D}_{0^{+}}^{\frac{3}{2}} \mathrm{v}(\sigma)=\frac{1}{\left(8+\sigma^{2}\right)(1+|\mathrm{v}(\sigma)|)}+\sigma, \quad \sigma \in \mathrm{J}, \\
\mathrm{v}(0)=0, \quad \mathrm{v}^{\prime}(0)=0 .
\end{array}\right.
$$

We conclude that $v$ satisfies (12). Therefore, we have

$$
|\mathrm{v}(\sigma)-\mathrm{u}(\sigma)| \leq \frac{\mu}{1-\mathcal{Q}_{\phi} \mu} e^{\sigma}=0.565276 e^{\sigma}, \quad \sigma \in \mathrm{J}
$$

see Fig. 3. 


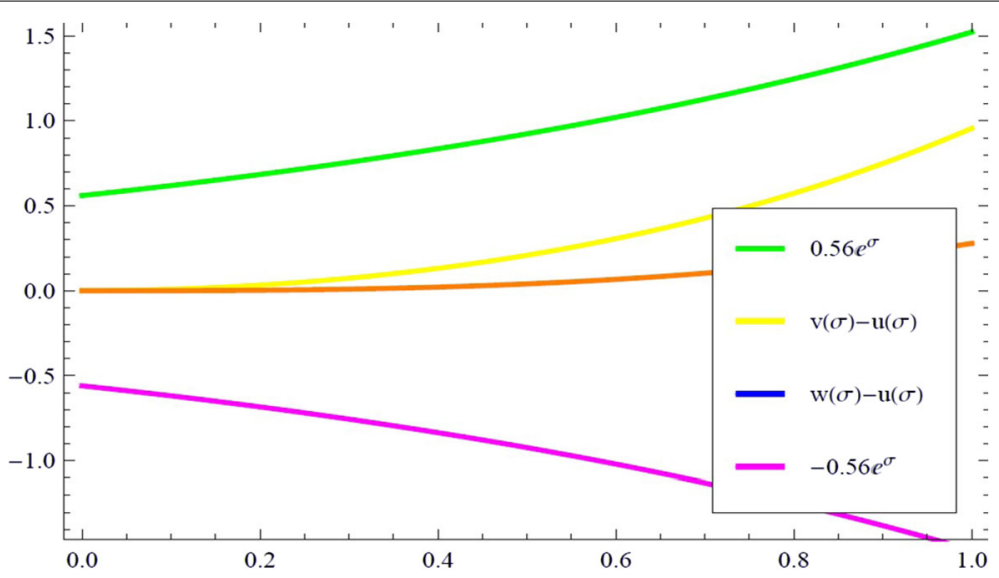

Figure 3 Functions $\mathrm{v}(\sigma)-\mathrm{u}(\sigma), \mathrm{w}(\sigma)-\mathrm{u}(\sigma)$, and $\pm 0.56 e^{\sigma}$

On the other hand, we consider $\mathrm{w} \in \mathrm{C}^{2}[0,1]$ as the solution of the following FDE:

$$
\left\{\begin{array}{l}
\mathrm{w}^{\prime \prime}(\sigma)+{ }^{c} \mathcal{D}_{0^{+}}^{\frac{3}{2}} \mathrm{~W}(\sigma)=\frac{1}{\left(8+\sigma^{2}\right)(1+|\mathrm{w}(\sigma)|)}+e^{\sigma}, \quad \sigma \in \mathrm{J}, \\
\mathrm{w}(0)=0, \quad \mathrm{w}^{\prime}(0)=0,
\end{array}\right.
$$

then w satisfies (12). Therefore, we have

$$
|\mathrm{w}(\sigma)-\mathrm{u}(\sigma)| \leq \frac{\mu}{1-\mathcal{Q}_{\phi} \mu} e^{\sigma}=0.565276 e^{\sigma}, \quad \sigma \in \mathrm{J}
$$

see Fig. 3.

\section{Conclusion}

Banach's contraction principle and Krasnoselskii's fixed point theorem have been successfully used in this work to accomplish the essential conditions for investigating the existence and uniqueness of solution of our proposed system. In this manner, under specific assumptions and conditions, the $\mathcal{H U S}$ and $\mathcal{H U R S}$ results have been demonstrated to study the solution of our proposed system. An illustrative example is given at the end to apply our theoretical results and show its validity. Some possible future directions of our work can be dedicated to applying our obtained results to study some interesting and important phenomena in physics and engineering such as elastic beam equation and fluid flow problems. Now possible extensions and generalizations of our obtained results can also be our future directions.

Acknowledgements

The second and sixth authors were supported by Azarbaijan Shahid Madani University. The authors express their gratitude to dear unknown referees for their helpful suggestions which improved the final version of this paper.

Funding

Not applicable.

Availability of data and materials

Data sharing not applicable to this article as no datasets were generated or analyzed during the current study.

Ethics approval and consent to participate

Not applicable. 


\section{Competing interests}

The authors declare that they have no competing interests.

\section{Consent for publication}

Not applicable.

\section{Authors' contributions}

The authors declare that the study was realized in collaboration with equal responsibility. All authors read and approved the final manuscript.

\section{Author details}

'Department of Mathematics, University of Peshawar, Peshawar, Khyber Pakhtunkhwa, Pakistan. ${ }^{2}$ Department of Exact Science and Engineering, 1 Decembrie 1918 University of Alba Iulia, Alba Iulia 510009, Romania. ${ }^{3}$ Department of Mathematics, Azarbaijan Shahid Madani University, Tabriz, Iran. ${ }^{4}$ Department of Medical Research, China Medical University Hospital, Taichung, Taiwan. ${ }^{5}$ Institute of Mathematical Sciences, University of Malaya, Kuala Lumpur 50603, Malaysia. ${ }^{6}$ Department of Mathematics and Statistics, Washington State University, Pullman, WA, USA.

\section{Publisher's Note}

Springer Nature remains neutral with regard to jurisdictional claims in published maps and institutional affiliations.

Received: 25 June 2021 Accepted: 30 July 2021 Published online: 21 August 2021

\section{References}

1. Rezapour, S., Imran, A., Hussain, A., Martinez, F., Etemad, S., Kaabar, M.K.A.: Condensing functions and approximate endpoint criterion for the existence analysis of quantum integro-difference FBVPs. Symmetry 13(3), 469 (2021). https://doi.org/10.3390/sym13030469

2. Alsaedi, A., Ahmad, B., Kirane, M., Torebek, B.: Blowing-up solutions of the time-fractional dispersive equations. Adv. Nonlinear Anal. 10(1), 952-971 (2021). https://doi.org/10.1515/anona-2020-0153

3. Guezane-Lakoud, A., Kiliçman, A.: On resonant mixed Caputo fractional differential equations. Bound. Value Probl. 2020, 168 (2020). https://doi.org/10.1186/s13661-020-01465-7

4. Liu, Y.: A new method for converting boundary value problems for impulsive fractional differential equations to integral equations and its applications. Adv. Nonlinear Anal. 8(1), 386-454 (2017). https://doi.org/10.1515/anona-2016-0064

5. Baleanu, D., Rezapour, S., Saberpour, Z.: On fractional integro-differential inclusions via the extended fractional Caputo-Fabrizio derivation. Bound. Value Probl. 2019, 79 (2019). https://doi.org/10.1186/S13661-019-1194-0

6. Abbas, M.l., Ragusa, M.A.: On the hybrid fractional differential equations with fractional proportional derivatives of a function with respect to a certain function. Symmetry 13(2), 264 (2021). https://doi.org/10.3390/sym13020264

7. Abbas, M.I., Ragusa, M.A.: Solvability of Langevin equations with two Hadamard fractional derivatives via Mittag-Leffler functions. Appl. Anal. (2021). https://doi.org/10.1080/00036811.2020.1839645

8. Alsaedi, A., Ahmad, B., Alghanmi, M.: Extremal solutions for generalized Caputo fractional differential equations with Stieltjes-type fractional integro-initial conditions. Appl. Math. Lett. 91, 113-120 (2019). https://doi.org/10.1016/j.aml.2018.12.006

9. Rezapour, S., Ntouyas, S.K., Iqbal, M.Q., Hussain, A., Etemad, S., Tariboon, J.: An analytical survey on the solutions of the generalized double-order $\phi$-integrodifferential equation. J. Funct. Spaces 2021, Article ID 6667757 (2021). https://doi.org/10.1155/2021/6667757

10. Tuan, N.H., Mohammadi, H., Rezapour, S.: A mathematical model for COVID-19 transmission by using the Caputo fractional derivative. Chaos Solitons Fractals 140, 110107 (2020). https://doi.org/10.1016/j.chaos.2020.110107

11. Baleanu, D., Mousalou, A., Rezapour, S.: A new method for investigating approximate solutions of some fractional integro-differential equations involving the Caputo-Fabrizio derivative. Adv. Differ. Equ. 2017, 51 (2017). https://doi.org/10.1186/S13662-017-1088-3

12. Baleanu, D., Mousalou, A., Rezapour, S.: On the existence of solutions for some infinite coefficient-symmetric Caputo-Fabrizio fractional integro-differential equations. Bound. Value Probl. 2017, 145 (2017). https://doi.org/10.1186/s13661-017-0867-9

13. Diethelm, K.A.: The Analysis of Fractional Differential Equations. Springer, Berlin (2010)

14. Granas, A., Dugundji, J.: Fixed Point Theory. Springer, New York (2003)

15. Hilfer, R:: Applications of Fractional Calculus in Physics. World Scientific, Singapore (2000)

16. Matar, M.M., Abbas, M.I., Alzabut, J., Kaabar, M.K.A., Etemad, S., Rezapour, S.: Investigation of the p-Laplacian nonperiodic nonlinear boundary value problem via generalized Caputo fractional derivatives. Adv. Differ. Equ. 2021, 68 (2021). https://doi.org/10.1186/s13662-021-03228-9

17. Ali, A., Shah, K., Abdeljawad, T., Mahariq, I., Rashdan, M.: Mathematical analysis of nonlinear integral boundary value problem of proportional delay implicit fractional differential equations with impulsive conditions. Bound. Value Probl. 2021, 7 (2021). https://doi.org/10.1186/s13661-021-01484-y

18. Ahmad, B., Ntouyas, S.K., Alsaedi, A., Alnahdi, M.: Existence theory for fractional-order neutral boundary value problems. Fract. Differ. Calc. 8(1), 111-126 (2018). https://doi.org/10.7153/fdc-2018-08-07

19. Aydogan, S.M., Baleanu, D., Mousalou, A., Rezapour, S.: On high order fractional integro-differential equations including the Caputo-Fabrizio derivative. Bound. Value Probl. 2018, 90 (2018). https://doi.org/10.1186/s13661-018-1008-9

20. Baleanu, D., Jajarmi, A., Mohammadi, H., Rezapour, S.: A new study on the mathematical modelling of human liver with Caputo-Fabrizio fractional derivative. Chaos Solitons Fractals 134, 109705 (2020).

https://doi.org/10.1016/j.chaos.2020.109705 
21. Baleanu, D., Etemad, S., Rezapour, S.: A hybrid Caputo fractional modeling for thermostat with hybrid boundary value conditions. Bound. Value Probl. 2020, 64 (2020). https://doi.org/10.1186/s13661-020-01361-0

22. Baleanu, D., Mohammadi, H., Rezapour, S.: Analysis of the model of HIV-1 infection of CD4(+) T-cell with a new approach of fractional derivative. Adv. Differ. Equ. 2020, 71 (2020). https://doi.org/10.1186/S13662-020-02544-W

23. Etemad, S., Ntouyas, S.K., Ahmad, B.: Existence theory for a fractional q-integro-difference equation with q-integral boundary conditions of different orders. Mathematics 7(8), 659 (2019). https://doi.org/10.3390/math7080659

24. Ntouyas, S.K., Etemad, S.: On the existence of solutions for fractional differential inclusions with sum and integral boundary conditions. Appl. Math. Comput. 266, 235-243 (2015). https://doi.org/10.1016/j.amc.2015.05.036

25. Ntouyas, S.K., Etemad, S., Tariboon, J.: Existence of solutions for fractional differential inclusions with integral boundary conditions. Bound. Value Probl. 2015, 92 (2015). https://doi.org/10.1186/s13661-015-0356-y

26. Etemad, S., Ntouyas, S.K.: Application of the fixed point theorems on the existence of solutions for q-fractional boundary value problems. AIMS Math. 4(3), 997-1018 (2019). https://doi.org/10.3934/math.2019.3.997

27. Rezapour, S., Mohammadi, H., Jajarmi, A.: A new mathematical model for Zika virus transmission. Adv. Differ. Equ. 2020, 589 (2020). https://doi.org/10.1186/S13662-020-03044-7

28. Ulam, S.M.: A Collection of Mathematical Problems. Interscience, New York (1968)

29. Hyers, D.H.: On the stability of the linear functional equation. Proc. Natl. Acad. Sci. 27(4), 222-224 (1941) https://doi.org/10.1073/pnas.27.4.222

30. Rassias, T.M.: On the stability of linear mappings in Banach spaces. Proc. Am. Math. Soc. 72(2), 297-300 (1978). https://doi.org/10.2307/2042795

31. Ali, Z., Kumam, P., Shah, K., Zada, A.: Investigation of Ulam stability results of a coupled system of nonlinear implicit fractional differential equations. Mathematics 7(4), 341 (2019). https://doi.org/10.3390/math7040341

32. Wang, J., Shah, K., Ali, A.: Existence and Hyers-Ulam stability of fractional nonlinear impulsive switched coupled evolution equations. Math. Methods Appl. Sci. 41(6), 2392-2402 (2018). https://doi.org/10.1002/mma.4748

33. Zada, A., Alam, M., Riaz, U.: Analysis of q-fractional implicit boundary value problem having Stieltjes integral conditions. Math. Methods Appl. Sci. 44(6), 4381-4413 (2021). https://doi.org/10.1002/mma.7038

34. Abbas, S., Benchohra, M., Lagreg, J.E., Alsaedi, A., Zhou, Y.: Existence and Ulam stability for fractional differential equations of Hilfer-Hadamard type. Adv. Differ. Equ. 2017, 180 (2017). https://doi.org/10.1186/s13662-017-1231-1

35. Chalishajar, D., Kumar, A.: Existence, uniqueness and Ulam's stability of solutions for a coupled system of fractional differential equations with integral boundary conditions. Mathematics 6(6), 96 (2018). https://doi.org/10.3390/math6060096

36. Vanterler da C. Sousa, J., Capelas de Oliveira, E.: Ulam-Hyers stability of a nonlinear fractional Volterra integro-differential equation. Appl. Math. Lett. 81, 50-56 (2018). https://doi.org/10.1016/j.aml.2018.01.016

37. Dai, Q., Gao, R., Li, Z., Wang, C.: Stability of Ulam-Hyers and Ulam-Hyers-Rassias for a class of fractional differential equations. Adv. Differ. Equ. 2020, 103 (2020). https://doi.org/10.1186/s13662-020-02558-4

38. Killbas, A.A., Srivastava, H.M., Trujillo, J.J.: Theory and Applications of Fractional Differential Equations. Elsevier, Amsterdam (2006)

39. Schiff, J.L.: The Laplace Transform: Theory and Applications. Springer, New York (1999)

40. Krasnoselskii, M.A.: Two remarks on the method of successive approximations. Usp. Mat. Nauk 10, 123-127 (1955)

41. Diaz, J.B., Margolis, B.: A fixed point theorem of the alternative for contractions on a generalized complete metric space. Bull. Am. Math. Soc. 74(2), 305-309 (1968)

\section{Submit your manuscript to a SpringerOpen ${ }^{\circ}$ journal and benefit from:}

- Convenient online submission

- Rigorous peer review

- Open access: articles freely available online

- High visibility within the field

- Retaining the copyright to your article

Submit your next manuscript at $\gg$ springeropen.com 